\title{
Correction to: Plumbagin Prevents IL-1 $\beta$-Induced Inflammatory Response in Human Osteoarthritis Chondrocytes and Prevents the Progression of Osteoarthritis in Mice
}

\author{
Wenhao Zheng, ${ }^{1}$ Zhenyu Tao, ${ }^{1}$ Chunhui Chen, ${ }^{1}$ Chuanxu Zhang, ${ }^{1}$ Hui Zhang, ${ }^{1}$ Xiaozhou Ying, \\ and Hua Chen ${ }^{1,2}$
}

\section{Correction to: Inflammation \\ https://doi.org/10.1007/s10753-017-0530-8}

The original version of this article contained mistakes, and the authors would like to correct them. The correct details are given below:

1. In Fig. 3c, the Western blotting pictures were used incorrectly. This is our mistake. The corrected Fig. $3 \mathrm{c}$ is shown in the next page.
The online version of the original article can be found at https://doi.org/ 10.1007/s10753-017-0530-8

\footnotetext{
${ }^{1}$ Department of Orthopaedic Surgery, The Second Affiliated Hospital and Yuying Children's Hospital of Wenzhou Medical University, 109 Xueyuan Xi Road, Wenzhou, 325000, China

${ }^{2}$ To whom correspondence should be addressed at Department of Orthopaedic Surgery, The Second Affiliated Hospital and Yuying Children's Hospital of Wenzhou Medical University, 109 Xueyuan Xi Road, Wenzhou, 325000, China. E-mail: chenhuafey@126.com
}

2. In Fig. 5, the immunofluorescence results of control group were used incorrectly. The corrected Fig. 5 is shown in the next page.

3. In Fig. 6a, the Western blotting of p-IKB was used incorrectly. The corrected Fig. 6a is shown in the next page.

The authors wish to apologize for these unintentional errors. 
a
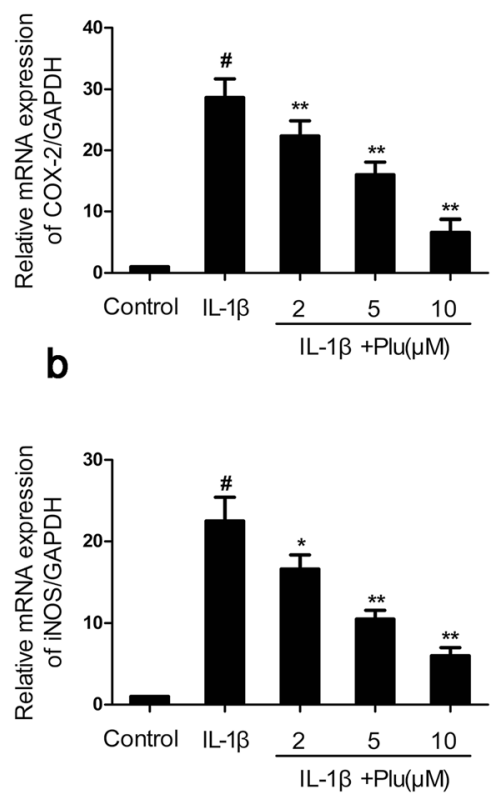

C

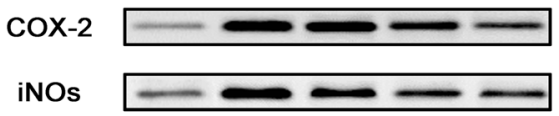

$\beta$-actin

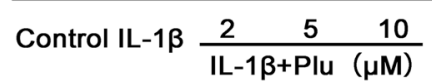

d
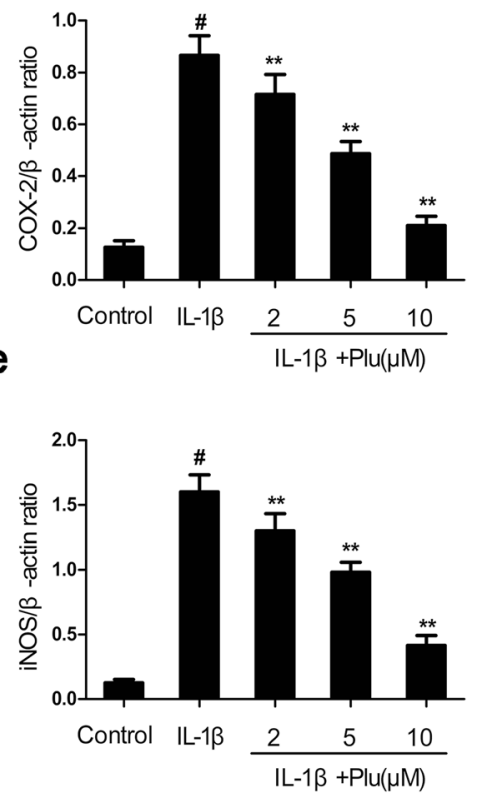

Fig. 3. Effect of plumbagin on IL-1 $\beta$-induced iNOs and COX-2 expression in human OA chondrocytes. Human OA chondrocytes were pretreated for $2 \mathrm{~h}$ with various concentrations of plumbagin $(2,5,10 \mu \mathrm{M})$ and then stimulated or not stimulated with IL-1 $\beta(10 \mathrm{ng} / \mathrm{ml})$ for $24 \mathrm{~h}$. ThemRNA expression levels of COX-2 (a) and iNOs (b) were assayed by qRT-PCR. The protein expression levels of iNOs and COX-2 were determined by Western blot and quantification analysis $(\mathbf{c}-\mathbf{e})$. The values are mean $\pm \mathrm{SD}$ of three independent experiments. $\# p<0.05$ compared with the control group; ${ }^{*} p<0.05, * * p<0.01$ compared with the IL-1 $\beta$ group. 


\section{Collagen-II}
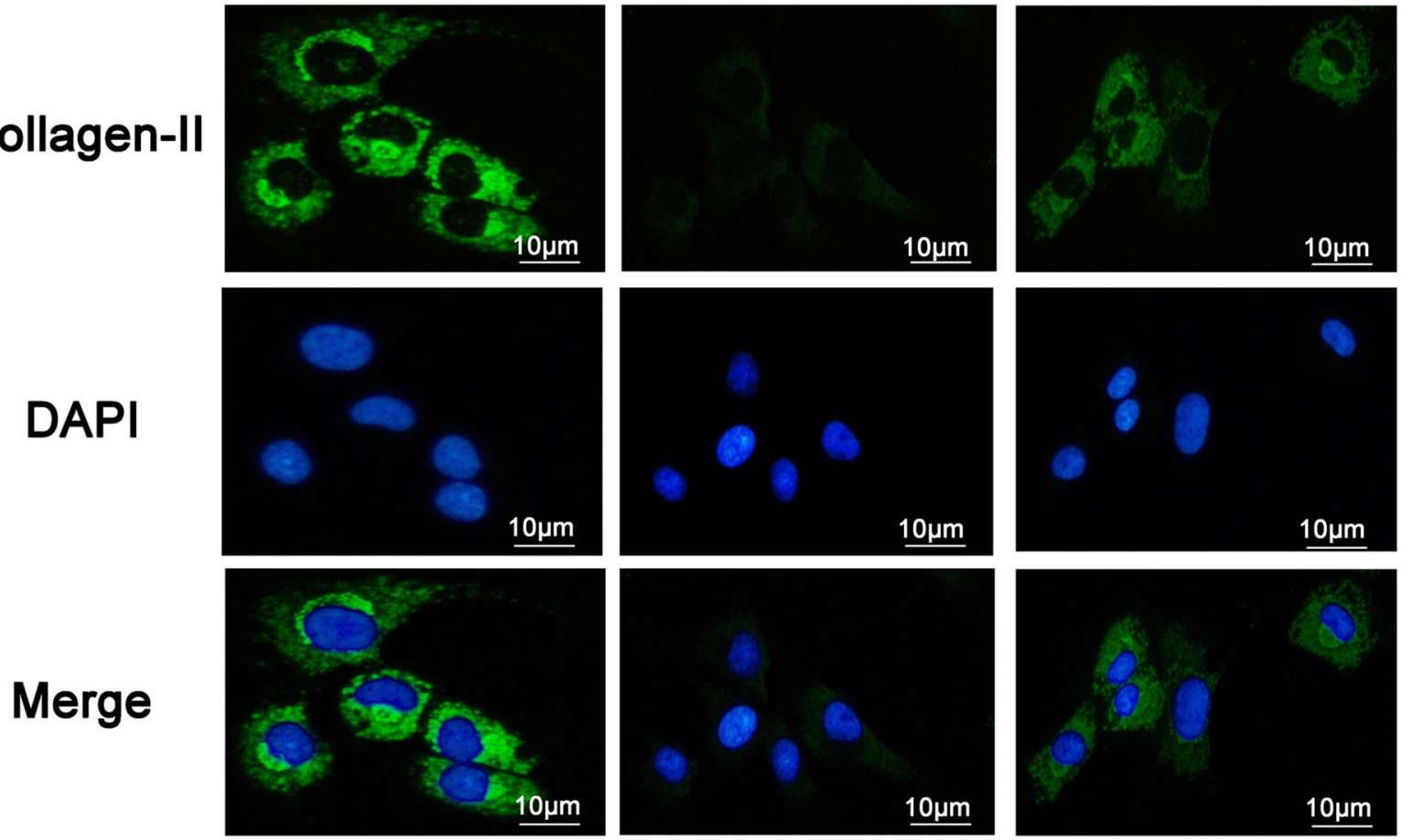

Control

IL-1 $\beta$

IL-1 $\beta+$ Plu

a

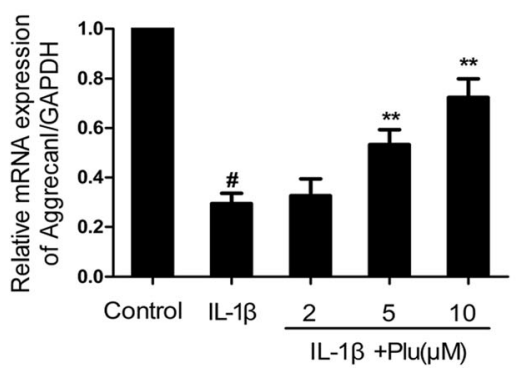

b

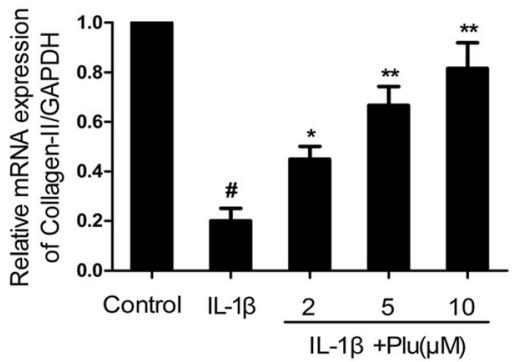

C

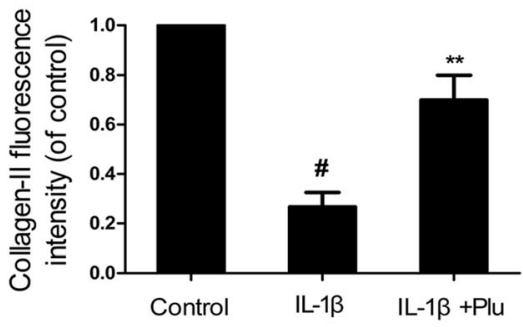

Fig. 5. Effect of plumbagin on IL-1 $\beta$-induced aggrecan and collagen-II degradation in human OA chondrocytes. Human OA chondrocytes were pretreated for $2 \mathrm{~h}$ with plumbagin $(2,5,10 \mu \mathrm{M})$ and then stimulated or not stimulated with IL-1 $\beta(10 \mathrm{ng} / \mathrm{ml}) \mathrm{for} 24 \mathrm{~h}$. The mRNA expression levels of aggrecan (a) and collagen-II (b) were assayed by qRT-PCR. The protein expression levels of collagen-II were determined by immunofluorescence and quantification analysis (c). The values are mean \pm SD of three independent experiments. $\# p<0.05$ compared with the control group; $* p<0.05, * * p<0.01$ compared with the IL- $1 \beta$ group. 


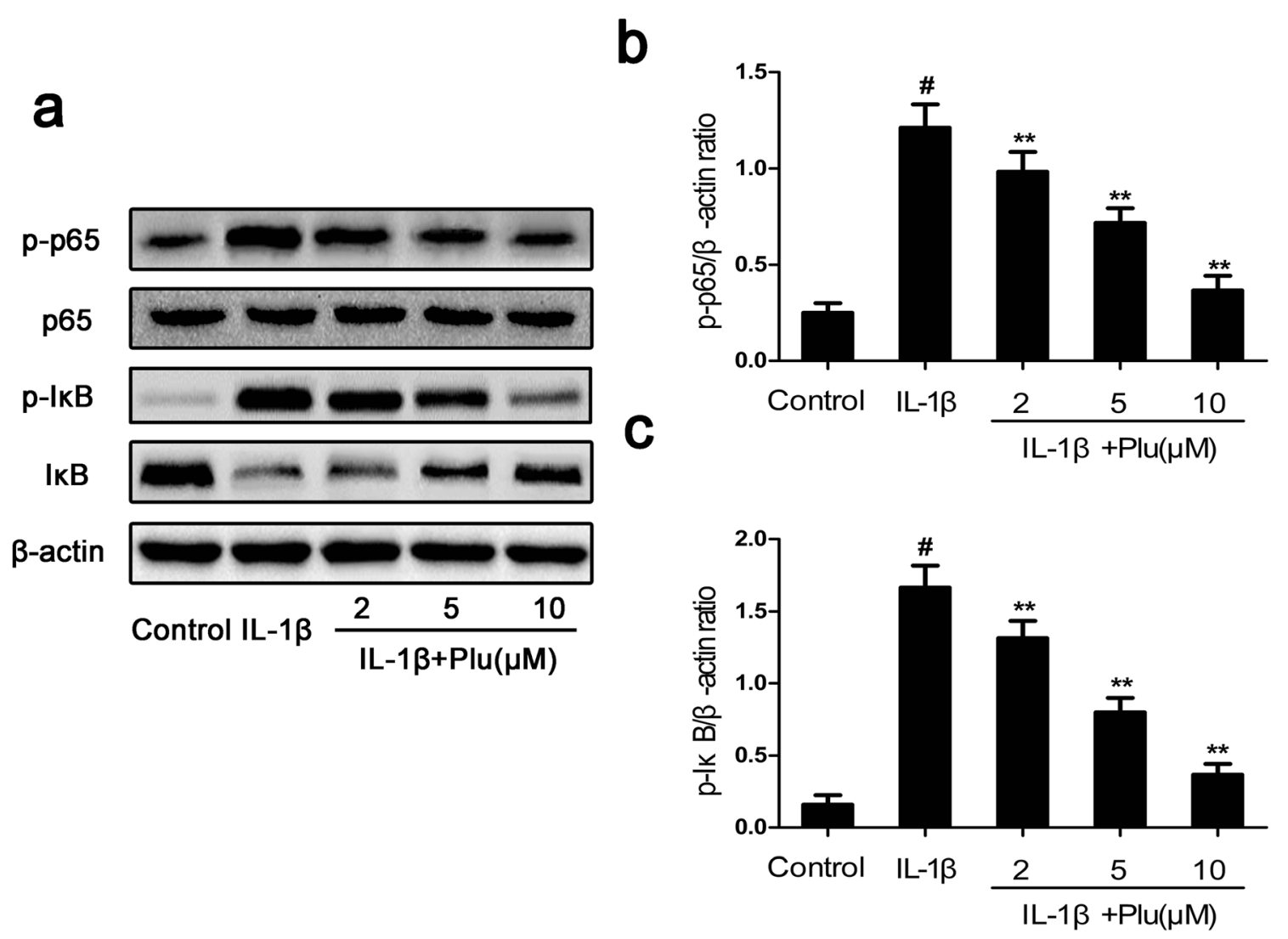

Fig. 6. Effect of plumbagin on IL-1 $\beta$-induced NF- $\mathrm{KB}$ activation in human OA chondrocytes. Chondrocytes were pretreated with plumbagin $(2,5,10 \mu \mathrm{M})$ for $2 \mathrm{~h}$, followed by stimulation with or without IL-1 $\beta(10 \mathrm{ng} / \mathrm{ml})$ for $1 \mathrm{~h}$. The protein expression levels of p65, p-p65, IkB, and p-IkB were determined by Western blot and quantification analysis $(\mathbf{a}-\mathbf{c})$. The values are mean $\pm \mathrm{SD}$ of three independent experiments. $\# p<0.05$ compared with the control group; $* p<0.05, * * p<0.01$ compared with the IL- $1 \beta$ group. 